\title{
The Treatabolome, an emerging concept
}

\author{
Gisèle Bonne \\ Sorbonne Université, Inserm, Institut de Myologie, Centre de Recherche en Myologie, F-75013 Paris, France \\ E-mail: g.bonne@institut-myologie.org
}

It is with a great pleasure that we introduce the first special issue of the Journal of Neuromuscular Diseases dedicated to the "Treatabolome", a new concept emerging from a European Project addressing unsolved rare diseases (Solve-RD, www.solverd.eu).

Thus, this special issue covers gene and variantspecific treatments for rare diseases, particularly rare neurological and neuromuscular disorders, thereby highlighting the important premise that a precise genetic diagnosis may result in equally precise therapeutic approaches. Although such targeted treatments are currently only available to a minority of RD patients, recent developments point towards a steep increase in the coming years, as suggested by the development of multiple gene therapies and the steady increase in the number of orphan drug applications.

One of the initial aims of the International Rare Diseases Research Consortium (IRDiRC), founded in 2011, was to contribute to the development of 200 new therapies for rare diseases by the year 2020, an objective reached ahead of schedule in 2016 [1]. They now propose 1000 new treatments by 2027 [2]. The need to increase the visibility of these treatments is the objective of the Treatabolome project within Solve-RD. This EU project aims at diagnosing unsolved rare disease cases and involves four European Reference Networks or ERNs (ERN-RND for Rare Neurological Diseases, ERN Euro-NMD for Rare Neuromuscular Diseases, ERN-ITHACA for rare congenital malformation and syndromes with intellectual and other neurodevelopmental disorders and ERN-GENTURIS for patients with one of the rare genetic tumour risk syndromes). Furthermore, it reaches out to the entire 24 ERNs networks and the undiagnosed disease programmes from Spain and Italy, following the ambitious goals set out by the
IRDiRC to diagnose and subsequently treat a representative number of rare diseases.

The growing availability of next generation genetic diagnostic tools is expanding the access to diagnosis even for patients that do not have access to primary expert evaluation. With this there is an evergrowing number of rare diseases' therapies are not necessarily "on the radar" of non-expert clinicians, who however increasingly take care and/or diagnose these patients. The constitution of a database of rare disease-specific treatments directly linked to the gene and variant level will allow flagging already available therapies at the time of diagnosis. For that purpose, the treatabolome database will be interoperable with different diagnosis-support tools and will complement their existing datasets with specific treatment information. The treatabolome data will be findable, available, interoperable and reusable (i.e., the FAIR principles of data management, [3]) to benefit rare disease patients and their clinicians and shorten the interval from diagnosis to treatment.

The Solve-RD project has invited expert teams to produce systematic literature reviews for rare diseases of their expertise and extract datasets of treatments for incorporation into the Treatabolome database. These systematic reviews shared a common structure, as detailed in a published methodology paper [4] and follow a pilot treatabolome publication on congenital myasthenic syndromes [5]. The treatabolome database incorporates these datasets, and an interoperable platform is under development to make available for users the data with existing clinical support, diagnostic tools and research systems, such as the RD-Connect's Genome Phenome Analysis Platform (https://rd-connect.eu).

The present 'Treatabolome' special issue of JND assembles five systematic reviews from prestigious reference centres representing a valuable 
concentration of rare diseases' expertise. In addition, we were also fortunate to be able to include in this special issue a review on the current therapeutic approaches in FSHD by Wang \& Tawil. It reviews the underlying disease mechanisms, potential therapeutic approaches and the state of trial readiness of future clinical trials in FSHD and thus is also treatabolome relevant as it highlights the therapeutic importance of making this genetic diagnosis, which is not always straightforward.

Over et al. from the Lübeck University and Hospital group managing the International Parkinson and Movement Disorder Society's database (MDSGene) focused their systematic literature review on therapies for Genetic Forms of Parkinson's Disease. They conclude that based on available data levodopa showed an overall good outcome, especially in LRRK2, VPS35, PARKIN, and PINK1 mutation carriers ("good" response in 94.6-100\%). They found that none of the available treatments is harmful to one genetic form but effective in another one, while they hope for novel disease-cause-modifying drugs.

The systematic review on therapies for Skeletal Muscle Ion Channelopathies by Desaphy et al. is a collaboration of two centres with a long-standing expertise on muscle channelopathies, from the University of Bari Aldo Moro (Bari, Italy) and from the Sorbonne Université-Inserm-Institute of Myology (Paris, France). They conclude that the treatment of these diseases is mainly symptomatic, aiming to reduce muscle excitability in non-dystrophic myotonias or modify triggers of periodic paralysis attacks on the other hand. They advocate performing more human studies to conceptualize and design mutationspecific precision medicine approaches in muscle ion channelopathies, an area that is still lagging behind.

The existing therapies for Hereditary Peripheral Neuropathies have been reviewed by Jennings et al. from the Cambridge Rare Diseases Group in the UK, with a contribution from the Institute of Myology in Paris. They collected all the evidence behind hereditary neuropathies' treatments. The stronger evidence links with treatments for TTR-related amyloid polyneuropathy, with significant evidence associated with therapies for several gene specific conditions, including CMT1, SPTLC1-related hereditary sensory neuropathy, Brown-Vialetto-Van Laere syndrome (SLC52A2/3) and Refsum disease (PHYH).

Manta et al. targeting the therapies for Metabolic Myopathies Related to Glycogen Storage and Lipid Metabolism, originates from Children's Hospital of Eastern Ontario Research Institute (Canada). They gather the evidence around specific treatments developed for metabolic myopathies (particularly enzyme replacement therapy with alglucosidase alfa for Pompe disease, riboflavin for multiple acyl-CoA dehydrogenase deficiency related to mutations in the ETFDH gene and carnitine for systemic primary carnitine deficiency). It is one area with significant potential growth in upcoming years, as more such specifically targeted treatments become available.

Finally, Atalaia et al. focuses on treatments for Laminopathies. This systematic review produced via a collaboration of French expert centres of the different types of Laminopathies summarizes the few existing treatments for the myriad of clinical manifestations arising from the LMNA gene. It highlights life-saving treatment recommendations around the cardiac manifestations as well as treatment approaches based on as of yet less developed evidence as it strives to condense all available knowledge about the subject. A major challenge this review highlights is the absence of specific genotype-phenotype pairings, as $L M N A$ is notorious for quite disparate phenotypical expressions, sometimes even concerning the same variant.

One of the next steps of the Treatabolome project is to extend it to rare diseases experts from all the 24 European Reference Networks and the whole scientific community producing other datasets to enrich the Treatabolome database and fulfil rare diseases' treatment awareness. This has been the goal of the treatabolome project from the its inception.

\section{ACKNOWLEDGMENTS}

The Solve-RD project has received funding from the European Union's Horizon 2020 research and innovation programme under grant agreement No 779257.

Gisèle Bonne

Sorbonne Université, France.

\section{REFERENCES}

[1] Dawkins HJS, Draghia-Akli R, Lasko P, Lau LPL, Jonker AH, Cutillo CM, Rath A, Boycott KM, Baynam G, Lochmüller H, Kaufmann P, Le Cam Y, Hivert V, Austin CP. International Rare Diseases Research Consortium (IRDiRC). Progress in Rare Diseases Research 2010-2016: An IRDiRC Perspective. Clin Transl Sci. 2018;11:11-20.

[2] Austin CP, Cutillo CM, Lau LPL,Jonker AH, Rath, A, Julkowska D, Thomson D, Terry SF, de Montleau B, Ardigò D, Hivert V, Boycott KM, Baynam G, Kaufmann P, Taruscio D, Lochmüller H, Suematsu M, Incerti C, Draghia-Akli 
R, Norstedt I, Wang L, Dawkins HJS. International Rare Diseases Research Consortium (IRDiRC) Future of Rare Diseases Research 2017-2027: An IRDiRC Perspective. Clin Transl Sci. 2018;11:21-27.

[3] Wilkinson MD, Dumontier M, Aalbersberg IJ, Appleton G, Axton M, Baak A. et al. The FAIR Guiding Principles for scientific data management and stewardship. Sci Data. 2016;3:160018.

[4] Atalaia A, Thompson R, Corvo A, Carmody L, Piscia D, Matalonga L. et al. A guide to writing systematic reviews of rare disease treatments to generate FAIR-compliant datasets: building a Treatabolome. Orphanet J Rare Dis. 2020;15(1):206.

[5] Thompson R, Bonne G, Missier P, Lochmuller H. Targeted therapies for congenital myasthenic syndromes: systematic review and steps towards a treatabolome. Emerg Top Life Sci. 2019;3(1):19-37. 